\title{
Clinical Indications for Intraocular Lens Power Calculation: A Prospective Randomised Study
}

\author{
S. M. THOMPSON and V. MOHAN-ROBERTS \\ West Bromwich
}

\begin{abstract}
Summary
Patients about to undergo cataract extraction were assessed clinically using several criteria to try and judge the necessity for biometry. The patients judged not to need biometry were allocated randomly to two groups, one of which had biometry.

The only significant clinical criterion for biometry was found to be the wearing of glasses since before the age of $\mathbf{3 0}$ years. Several unexpectedly high refractive errors occurred in the group of patients who did not have biometry. In the group of patients judged not to need biometry but allocated to the biometry group, refractive results were significantly better (nearer to target refractions) than in the unmeasured group.

Routine pre-operative biometry is probably the only way to avoid unexpected high ametropia, and it also improves the refractive results. There is however much room for improvement in the accuracy of biometry.
\end{abstract}

The necessity for routine measurement of the eye and calculation of intraocular lens power ('biometry') before cataract extraction with intraocular lens implantation is still debated. There has been some support for the contention that routine biometry is not necessary for patients with small refractive errors. ${ }^{1,2}$ Our previous study? . showed no significant difference in the refractive results between a group of patients who had biometry and one who did not. In the present study patients judged clinically to be at risk of ametropia were selected for biometry before cataract surgery. The remaining patients were randomised into two groups, one of which had biometry.

The study was designed to answer three questions. First, is it possible clinically to identify patients at risk of post-operative high ametropia if a standard lens were implanted? Secondly, if so, which clinical characteristics are significant? Thirdly, if patients are judged clinically to need biometry does it in fact significantly improve the refractive result?

\section{Method}

All the authors' patients who were listed for cataract surgery were examined to assess the need for biometry. Criteria for biometry were the wearing of glasses since before the age of 30 years; hypermetropia or myopia of three dioptres or more but glasses worn only from the age of thirty; clinical signs suggesting myopia or hypermetropia such as deep or shallow anterior chambers; amblyopia; anisometropia or squint. These are similar to criteria suggested by Percival. ${ }^{3}$ This group of patients was called group $\mathbf{A}$.

The remaining patients were allocated by means of a random numbers table to measured and unmeasured groups (group 
Table I. Clinical criteria for biometry (group $A$ ).

\begin{tabular}{lr}
\hline Glasses since before the age of thirty: & 40 \\
Myopic refraction: & 21 \\
Hypermetropic refraction: & 16 \\
No refraction recorded: & 3 \\
Glasses since the age of thirty or over & 21 \\
(refraction 3D or more): & 16 \\
Myopic correction: & 5 \\
hypermetropic correction: & 8 \\
Fellow eye ametropic after implantation & 3 \\
of a standard lens: & 2 \\
Deep anterior chambers: & 1 \\
Amblyopia: & 1 \\
"Myopic discs": & \\
Age under forty: & \\
\hline
\end{tabular}

B-M and group B-UM). All patients having biometry underwent keratometry with a Haag-Streit keratometer. Before December 1986 axial lengths were measured with a Radionics Echo-Oculometer 3000, and the intraocular lens powers required for emmetropia and one dioptre of myopia were calculated using the Binkhorst formula. After that time axial lengths were measured with a Cooper Vision Ultrascan Digital B System 1V and the intraocular lens powers required to produce emmetropia and a wide range of other refractions were calculated using the SRK formula, with A constants recommended by the lens manufacturers. During 1987 the surgical method was changed from intracapsular extraction with a Binkhorst iris clip lens to extracapsular extraction with a Sinskey-style posterior chamber lens.

After biometry a lens designed to produce a post-operative refraction between -1.0 dioptre and emmetropia was usually chosen. In cases of high ametropia a post-operative refraction within four dioptres of the fellow eye was usually chosen.

Patients in the unmeasured group received a standard power lens (19 or 19.5 dioptre iris clip, or 20 or 21 dioptre posterior chamber)

Each patient was refracted at least six weeks after surgery, and the refraction recorded as the spherical equivalent.

Those patients whose final refraction was known at the time of termination of the study comprised the study groups. Data was analysed statistically using the t-test, the Mann-Whitney $U$ test and the variance ratio test.

\section{Results}

\section{(1) Patients judged clinically to need biometry (Group A).}

There were sixty-four patients in this group. Another twelve patients underwent biometry but were excluded from the final group. The reasons for exclusion were (a) posterior capsule rupture and insertion of a standard power anterior chamber lens (five patients). Standard power lenses were used because a range of powers was not available. (b) high myopia: no intra-ocular lens used (four patients). (c) no post-operative refraction available (three patients).

All seventy-six patients were included in the analysis of reasons for biometry, which are shown in Table 1.

Figure 1 shows the range of lenses calculated to produce emmetropia in the 76 patients judged to need biometry. This can be compared with the range needed for emmetropia by patients judged not to need biometry (Group B-M, Fig 7). Using the method of normal scores the B-M measurements can be shown to be consistent with having come from a normal distribution. The mean lens powers for emmetropia (18.8D posterior chamber and $18.5 \mathrm{D}$ iris clip) are close to published reports of the range for large unselected series of cataract patients $\mathrm{s}^{4,5}$ Group B-M can therefore act as a control for

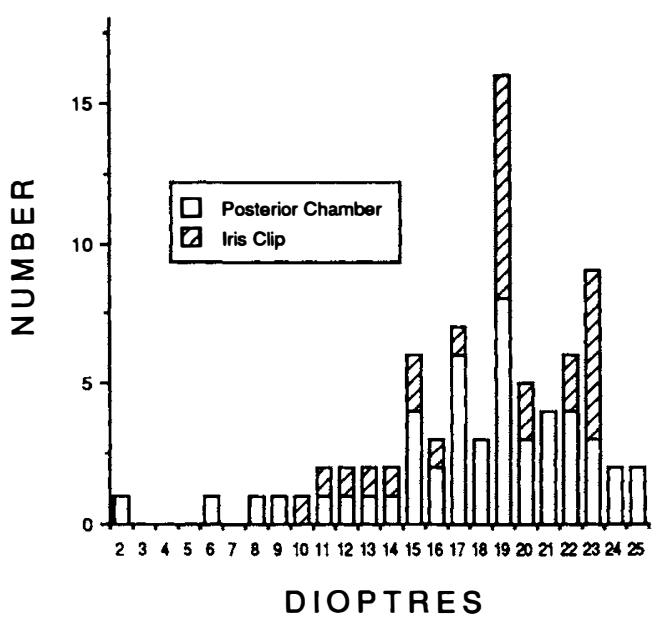

Fig. 1. Range of lenses calculated to produce emmetropia in patients judged clinically to need biometry (group $A$ ). 

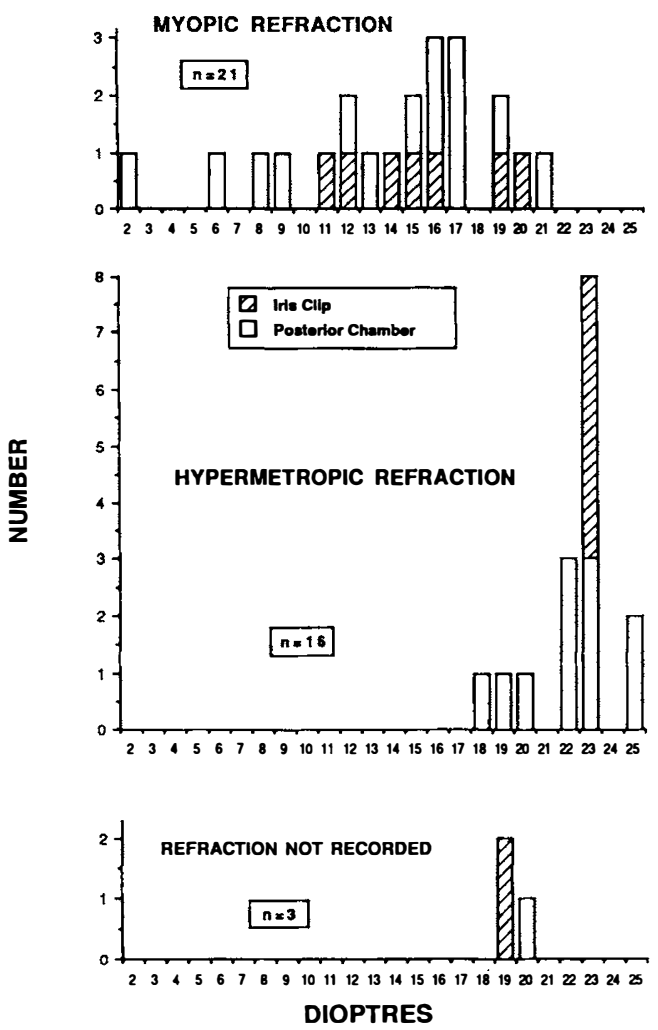

Fig. 2. Range of lenses calculated to produce emmetropia in group A patients wearing glasses since before the age of thirty.

group A. The median power of lenses required for emmetropia in group A was 19 dioptres (posterior chamber and iris clip), which is the same as the median for group B-M, and therefore not significantly different (Mann-Whitney, $\mathrm{p}=0.86$ posterior chamber, $\mathrm{p}=0.92$ iris clip) However the (statistical) variance of powers within group A differs significantly from that of group B-M (variance ratio test, $P=<0.005$, iris clip. The distribution of posterior chamber lenses within group A was not normal and therefore a variance ratio test could not be used). This implies that group A came from a different population than group B-M, as would be expected if clinical judgment successfully identified patients with ametropia. These results suggest but do not prove that it is possible to identify patients who need biometry on clinical grounds.

The most common clinical reason for bio- metry was the wearing of glasses since before the age of thirty. There were 40 people in this category; 21 of whom had a myopic correction (range -1.50 to $-14.0 \mathrm{D}$, spherical equivalent), 16 a hypermetropic correction (range +1.50 to $+4.0 \mathrm{D}$ ), and three whose pre-operative refraction was not recorded. The lens powers calculated to produce emmetropia in these patients are shown in Figure 2. The median lens power required by the myopic group was 15 dioptres (posterior chamber and iris clip lenses). This was significantly less than the median lens power for group B-M (19 dioptres, $p=0.01$, Mann-Whitney). The median lens powers for the hypermetropic group were 22 dioptres (posterior chamber) and 23 dioptres (iris clip). These were significantly higher than the medians for group B-M $(\mathrm{p}=0.0003$ for posterior chamber lenses and $\mathrm{p}=0.0006$ for iris clip lenses, Mann-Whitney). Also, only four people in these two groups would have been expected to achieve emmetropia with a standard power lens. This suggests that the wearing of glasses since

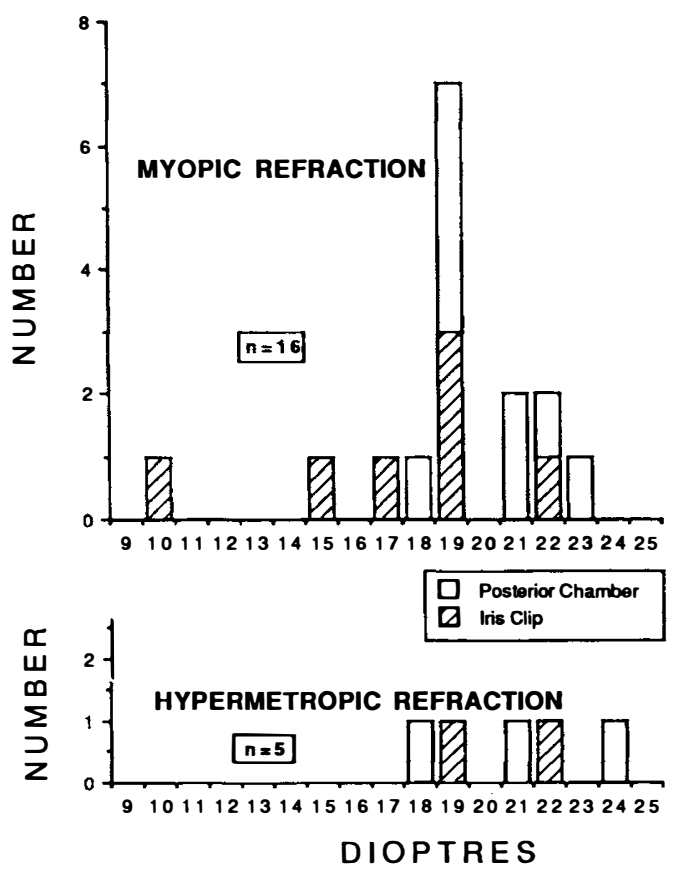

Fig. 3. Range of lenses calculated to produce emmetropia in group $A$ patients wearing glasses since the age of thirty or over, with pre-operative refraction $3 D$ or more. 


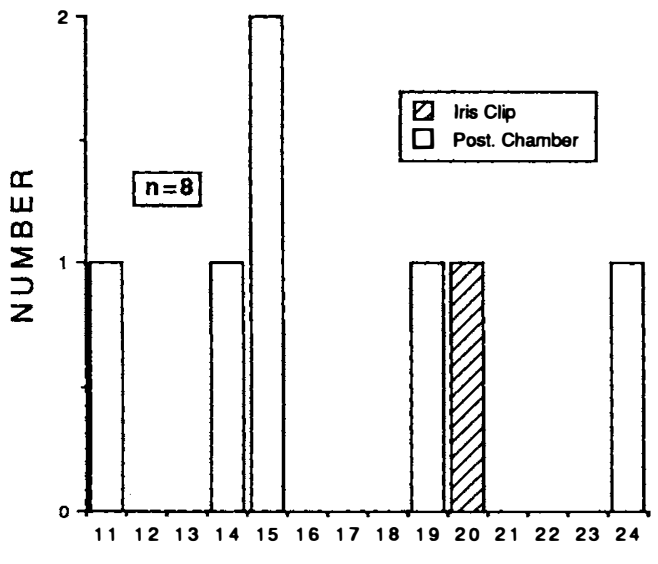

DIOPTRES

Fig. 4. Range of lenses calculated to produce emmetropia in group $A$ patients with ametropia in the fellow pseudophakic eye.

before the age of thirty is a useful clinical indication of biometry. The group whose preoperative refractions were unknown is too small for analysis.

Figure 3 illustrates the next most common reason for biometry, the wearing of distance glasses since the age of 30 years with a refractive error of three dioptres or more. There were sixteen patients with a myopic correction (range -3.0 to $-4.0 \mathrm{D}$, spherical equivalent), and five patients with a hypermetropic correction (range +3.0 to $+4.0 \mathrm{D}$ ). The median lens power required for emmetropia by the myopic group was 19 dioptres for both the posterior chamber and iris clip groups. This is no different from the median for group B-M. However, one patient would have needed a 10 dioptre lens and one a 15 dioptre lens to achieve emmetropia, indicating high myopia. Another was calculated to need a 23 dioptre lens indicating unexpected hypermetropia, and perhaps illustrating the well-known myopic shift which can occur with nuclear sclerosis.

The median lens power required for emmetropia by the hypermetropic group was 21 dioptres (posterior chamber) and this does not differ significantly from the group B-M median $(p=0.16$, Mann-Whitney). The number of iris clip lenses calculated for this group was too small for analysis but shows a trend towards the need for higher power lenses as would be expected.

These results suggest that although a refractive error of 3 dioptres or more, if glasses have been worn only since the age of thirty may indicate ametropia, it is not a reliable clinical indication for biometry.

Figure 4 shows the range of lenses required for emmetropia in the group of eight patients whose clinical indication for biometry was that a standard lens implanted in the other eye resulted in ametropia ranging from -5.0 to $+5.0 \mathrm{D}$. The number of iris clip lenses is too small for analysis, but for posterior chamber lenses the median lens power calculated to produce emmetropia was 15 dioptres. This was not significantly different from the group B-M median ( $\mathrm{p}=0.11$, Mann-Whitney). This suggests that ametropia in the fellow pseudophakic eye is not a statistically reliable reason for biometry, although it may still be judged to be clinically significant.

The remaining four reasons for biometry are shown in Figure 5. The small group of patients with deep anterior chambers as an indication for biometry show a trend towards lenses weaker than the mean, as would be expected in myopes. The numbers are too small for statistical analysis.

The characteristics of the 64 patients in the post-operative study group $\mathrm{A}$ are shown in Tables II-IV. They do not differ significantly from group B patients in sex, length of time after surgery to final refraction, cylindrical

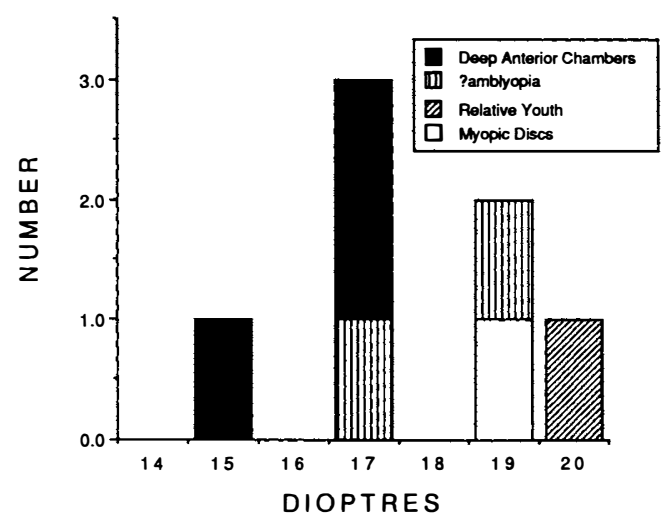

Fig. 5. Range of lenses calculated to produce emmetropia in group A patients with other indications for biometry. 
Table II. Characteristics of study groups-age, sex, and length of time to refraction.

\begin{tabular}{|c|c|c|c|c|c|c|c|}
\hline \multirow[b]{2}{*}{ Group } & \multirow[b]{2}{*}{ No. } & \multicolumn{2}{|c|}{ Age (years) } & \multicolumn{2}{|c|}{ Sex } & \multicolumn{2}{|c|}{$\begin{array}{l}\text { Length of time to } \\
\text { refraction (weeks) }\end{array}$} \\
\hline & & Mean & $S D$ & $M$ & $F$ & Mean & $S D$ \\
\hline$A$ & 64 & 68.83 & 9.38 & 22 & 42 & 13.89 & 9.96 \\
\hline$B-M$ & 61 & 70.43 & 8.68 & 27 & 35 & 16.20 & 9.92 \\
\hline$B-U M$ & 62 & 73.53 & 8.20 & 28 & 34 & 14.56 & 9.25 \\
\hline
\end{tabular}

correction or type of lens (posterior chamber or iris clip) used. Group A was significantly older than group $\mathrm{B}$.

Figure 6 shows the difference between the predicted and post-operative refractions in group A. In this group $51.6 \%$ of post-operative refractions fell within one dioptre of the expected result, $90.6 \%$ within two dioptres, and $96.9 \%$ within three dioptres.

\section{(2) Patients judged clinically not to need biometry, and randomised into measured (Group B-M) and unmeasured (Group $B$-UM) groups.}

There were sixty-one patients in group B-M and sixty-two in group B-UM. The characteristics of each group are shown in Tables II-IV. There is no significant difference between the groups in sex, length of time after surgery to final refraction, cylindrical correction or type of lens used (posterior chamber or iris clip). Group B-M is significantly younger than group B-UM $(p=0.044$, t-test $)$.

The range of lenses calculated to produce emmetropia in group B-M is shown in Figure 7. For seven $(11 \%)$ patients in group B-M the lens calculated to produce emmetropia was four dioptres or more different from the standard lens power. Use of a standard lens in these patients would be expected to produce a refractive error more than two dioptres from emmetropia, which had not been predicted clinically.

Figure 8 shows the difference between the predicted and actual post-operative refractions for group B-M. $63.9 \%$ fall within one dioptre of the target, $91.8 \%$ within two dioptres, and $100 \%$ within three dioptres.

The range of lenses used in the unmeasured group B-UM is shown in Table V.

Figure 9 shows the difference between emmetropia (the implied target) and post- operative refractions for group B-UM. 30.6\% fall within one dioptre of the target, $67.7 \%$ within two dioptres, and $87 \%$ within three dioptres. Eight patients $(12.9 \%)$ had postoperative spherical equivalents more than three dioptres from emmetropia. There were three unexpected high refractive errors of $-6.0,-7.5$, and -8.75 dioptres.

The difference between the predicted and actual post-operative refractions of the measured and unmeasured groups is highly statistically significant $(\mathrm{p}=<0.00005$, $\mathrm{t}$-test $)$. If the refractive results of the 18 measured (B-M) patients who were calculated to achieve emmetropia are compared with group B-UM (where the implicit target was emmetropia), the group B-M patients are still significantly closer to the target $(p=0.008$, t-test). This implies that the chances of producing a post-operative refraction close to the desired result are better if biometry is per-

Table III. Characteristics of study groups-post-operative astigmatism.

\begin{tabular}{lccc}
\hline & \multicolumn{3}{c}{ Cylindrical error (dioptres) } \\
\cline { 2 - 4 } & Range & mean & $S D$ \\
\hline$A$ & $0-4.5$ & 1.57 & 1.12 \\
$B-M$ & $0-5.0$ & 1.91 & 1.13 \\
$B-U M$ & $0-4.5$ & 1.88 & 1.16 \\
\hline
\end{tabular}

Table IV. Characteristics of study groups-types of lens used.

\begin{tabular}{lcc}
\hline & \multicolumn{2}{c}{ Type of lens used } \\
\cline { 2 - 3 } Group & $\begin{array}{c}\text { Posterior } \\
\text { chamber }\end{array}$ & Iris clip \\
\hline$A$ & 42 & 22 \\
$B-M$ & 35 & 26 \\
$B-U M$ & 36 & 26 \\
\hline
\end{tabular}




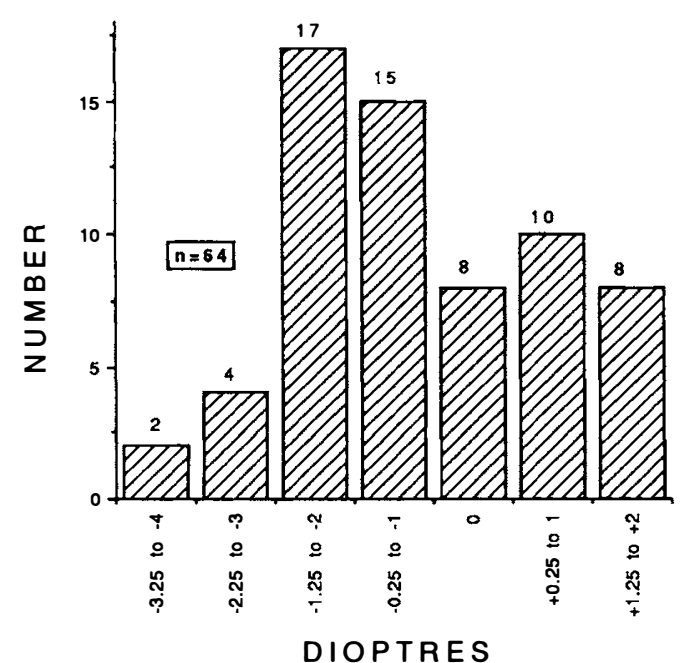

Fig. 6. Group A: Difference between predicted and post-operative refractions.

formed, even if patients judged clinically to need biometry are excluded first.

\section{Discussion}

The main reasons for routine pre-operative biometry are to exclude unexpected high ametropia, and to achieve a desired post-operative refraction. In 1986 we published a study in which the refractive results for a group of patients who had pre-operative biometry were compared with those of a group who had standard lenses implanted. ${ }^{2}$ There were no unexpected high refractive errors in the unmeasured group and the refractive results of the two groups did not differ significantly. However the number of patients in each group was small, and unexpected high ametropia has occurred in other series. ${ }^{5,6,7}$ Also, the patients undergoing biometry were not selected randomly, which may have meant that people known to be ametropic were placed in the measured group. This would tend to reduce the post-operative difference between the groups.

We therefore did a randomised study with larger numbers of patients to try and decide whether it is possible to identify those at risk of high ametropia, and if routine biometry is of benefit for the remaining patients.

The results suggest that although it is often possible to judge clinically that patients need biometry, unexpected high refractive errors still occur in some patients judged not to need biometry. In practice in the United Kingdom, although implantation of intraocular lenses after cataract removal is common, ${ }^{8}$ routine biometry is not always done. The results of our study suggest that if, despite the risk of unexpected high ametropia, clinical judgment to select for biometry is relied on, the only reliable clinical consideration likely to identify patients with high refractive errors is a history of glasses since before the age of thirty years.

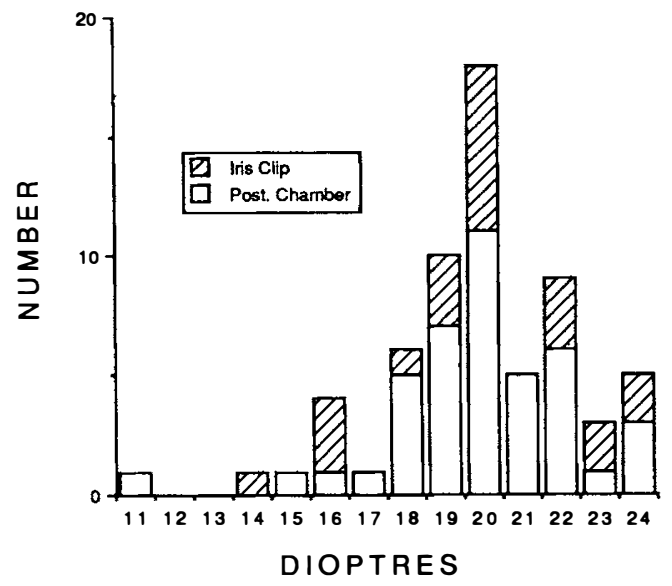

Fig. 7. Range of lenses calculated to produce emmetropia in patients judged clinically not to need biometry (group $B-M$ ).

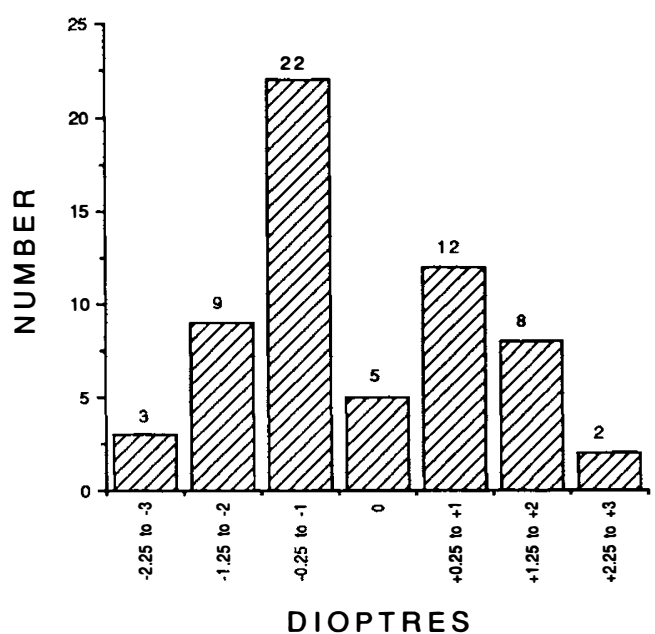

Fig. 8. Difference between predicted and post-operative refractions, measured patients (group B-M). 
Table V. Lenses used in group B-UM.

\begin{tabular}{lccc}
\hline & $19-19.5 D$ & $20-20.5 D$ & $21 D$ \\
\hline $\begin{array}{l}\text { Posterior } \\
\text { chamber }\end{array}$ & - & 26 & 8 \\
\begin{tabular}{l} 
Iris clip \\
\hline
\end{tabular} & 26 & - & - \\
\hline
\end{tabular}

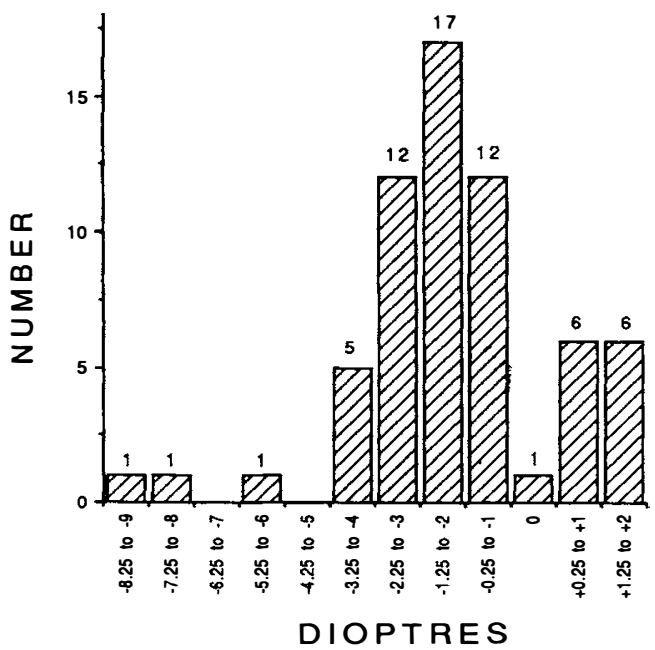

Fig. 9. Difference between post-operative refractions and emmetropia, unmeasured patients (group B-UM).

Singh et al., ${ }^{1}$ have questioned the need for routine biometry in people with pre-operative refractive errors of less than three dioptres. They have suggested that the use of standard power IOLs would give satisfactory results in practice. Our previous study supports this contention. However Singh et al were estimating refractive results if standard power IOLs had been used; the present study shows the results if standard power lenses are actually used. The post-operative refractions of patients who had pre-operative biometry were significantly closer to the desired result than those implanted with standard power IOLs.

However even if pre-operative biometry was performed, its accuracy in this series does not compare well with some others reported, ${ }^{7,8}$ although a similar degree of inaccuracy has been reported by other workers. ${ }^{9}$ The accuracy of biometry can be improved by altering the $\mathrm{A}$ constant, ${ }^{10,11}$ and this may have helped in our series. Another source of error is inaccurate measurement of axial length. ${ }^{7,11}$ The formula may also need to be modified to improve accuracy for very short or long eyes. ${ }^{11}$

A further cause for concern is post-operative astigmatism. In our series several patients had cylindrical errors of three, four or five dioptres, and other workers have reported similar astigmatic errors. ${ }^{1,13}$. High astigmatism negates one purpose of biometry which is to give the patient satisfactory vision without glasses. Although the results of this study support the use of routine biometry, it would be of even more value if its accuracy could be improved, and post-operative astigmatism controlled.

\section{Conclusion}

The results of this study suggest some answers to the questions posed. First it is possible to identify pre-operatively some but not all patients at risk of high ametropia. Secondly, the only clinical characteristic which identifies these patients significantly more often than chance is the wearing of glasses since before the age of thirty years. Thirdly, refractive outcomes are improved by routine biometry even for patients with no clinical indication for biometry.

Routine biometry would be even more valuable if its accuracy were improved, and postoperative astigmatism controlled.

We thank Tim Marshall for statistical advice and Alastair Rose for the illustrations.

\section{References}

${ }^{1}$ Singh K, Sommer A, Jensen AD, Payne JW. Intraocular lens power calculations. A practical evaluation in normal subjects at the Wilmer Institute. Arch Ophthalmol 1987, 105: 1046-50.

${ }^{2}$ Thompson SM and Mohan-Roberts V. A comparison of post-operative results with and without intraocular lens power calculation. $\mathrm{Br} J$ Ophthalmol 1986, 70: 22-5.

${ }^{3}$ Percival P. Lens power calculation-is it necessary? Trans Ophthalmol Soc UK 1983, 103: 577-9.

${ }^{4}$ Hillman JS. The selection of intraocular lens power by calculation and by reference to the refraction-a clinical study. Trans Ophthalmol Soc UK 1982, 102: 495-7.

${ }^{5}$ Gregory PTS, Esbester RM, Boase DL. Accuracy of routine intraocular lens calculation in a district general hospital. $\mathrm{Br} J$ Ophthalmol 1989, 73: 57-60.

${ }^{6}$ Olsen T. Pre- and post-operative refraction with 
implantation of standard power IOL. $\mathrm{Br} J$ Ophthalmol 1988, 72: 231-5.

${ }^{7}$ Halliday BL. Calculation of intraocular lens power-results in practice. Trans Ophthalmol Soc UK 1986, 105: 435-40.

${ }^{8}$ Wong D and Steele ADMcG. A survey of intraocular lens implantation in the United Kingdom. Trans Ophthalmol Soc UK 1985, 104: 760-5.

${ }^{9}$ Hoffer KJ. Accuracy of intraocular lens calculation. Arch Ophthalmol 1981, 99: 1819-23.

${ }^{10}$ Singh $M$ and Dahalan A. Significance of intraocular lens power calculation. BrJ Ophthalmol 1987, 71: 850-3.
${ }^{11}$ Holladay JT, Prager TC, Ruiz RS, Lewis JW, Rosenthal $\mathrm{H}$. Improving the predictability of intraocular lens power calculations. Arch Ophthalmol 1986, 104: 539-41.

${ }^{12}$ Menezo JL, Chaques V, Harto M. The SRK regression formula in calculating the dioptric power of intraocular lenses. $\mathrm{Br} J$ Ophthalmol 1984, 68: 235-7.

${ }^{13}$ Hillman JS. The computer calculation of intraocular lens power. A clinical study. Trans Ophthalmol Soc UK 1980, 100: 222-8. 